\title{
Calophyllum inophyllum, eine wenig bekannte Öl-Pflanze
}

\author{
HERW IG ZaHORKA
}

\begin{abstract}
Indonesia initiated a biodiesel pilot project on the island of Madura in March of 2010. There, the endemic tree Calophyllum inophyllum (indon. Nyamplung) is being cultivated on a surface of about 10000 ha along the coasts. The seeds contain 50 $74 \%$ oil, which can be processed into biodiesel. Test vehicles have performed successfully over a distance of several thousand kilometers. Production costs have been calculated to be 4000 RP (about 30 euro cents) per liter. The article features a description of the tree and its potential economic utility.
\end{abstract}

\section{Zusammenfassung}

Indonesien begann im März 2010 mit einem Bio-Diesel Pilotprojekt auf der Insel Madura. Auf 10000 ha wird der dort an den Küsten heimische Baum Calophyllum inophyllum (indon. Nyamplung) angebaut. Die Samen enthalten 50-74\% Öl, das zu BioDiesel verarbeitet werden kann. Probefahrten mit diesem Treibstoff über einige $1000 \mathrm{~km}$ verliefen erfolgreich. Die Erzeugerkosten wurden bisher mit 4000 RP (etwa 30 Cent) je Liter berechnet. Der Baum und seine Nutzungsmöglichkeiten werden vorgestellt.

\section{Baum mit vielen Namen}

Die Gattung Calophyllum (Calophyllaceae) umfasst etwa 190 Arten. Calophyllum inophyllum hat eine weite geografische Verbreitung von Ostafrika über Madagaskar, Indien, Indochina, Thailand, Philippinen, Malaysia, Indonesien bis nach Nordaustralien. Er kommt auch auf einigen Inseln im Pazifik vor. Der tropische Tieflandsbaum gedeiht an sandigen Stränden oder auch in höheren Lagen bis $500 \mathrm{~m}$. Aufgrund seiner weiten Verbreitung gibt es für ihn zahlreiche lokale Bezeichnungen. Der wissenschaftliche Name leitet sich von gr. kalos = schön und phyllon = Blatt ab. Er hat u.a. die Bezeichnungen Ball nut tree, Calaba tree, Alexandrian laurel, Tamanu tree, Borneo mahogany, Nyamplung, Bintangor laut, Penaga oder Palo mariae.

\section{2. Äußeres Erscheinungsbild}

Calophyllum inophyllum ist ein mittelgroßer, langsam wachsender Baum, der selten über $25 \mathrm{~m}$ hoch wird. Er hat tief reichende Wurzeln und neigt nicht zu Brettwurzeln. Der Baum kann einen Stammdurchmesser bis über $1 \mathrm{~m}$ erreichen. Typisch sind die unregelmäßig verzweigten Äste, die breite Krone und eine dichte immergrüne Belaubung. Die längsrissige Borke ist grau bis braun gefärbt. Die Furchen vertiefen sich im Al-

Abb. 1: Blüten und Knospen von Calophyllum inophyllum. ter und gehen dann teilweise ineinander über. Bei Verletzungen erscheint reichlich braungelber bis rötlicher Milchsaft, der klebrig und giftig ist. Die Borke ist dick und faserig. Ihre im Innern rosa bis rote Farbe dunkelt an der Luft bräunlich nach. Das Splintholz ist gelbbraun bis hellrosa. Es ist undeutlich vom rotbraunen, rosabraunen oder orangebraunen Kernholz abgesetzt. Seine Textur ist ungleichmäßig und oft grob. Eine polierte Oberfläche erscheint glänzend. Die relativ weitlumigen Gefäße sind im Holzquerschnitt mit bloßem Auge sichtbar. Meist sind sie einzeln, können aber auch in ungleichmäßigen radialen Ketten angeordnet sein.

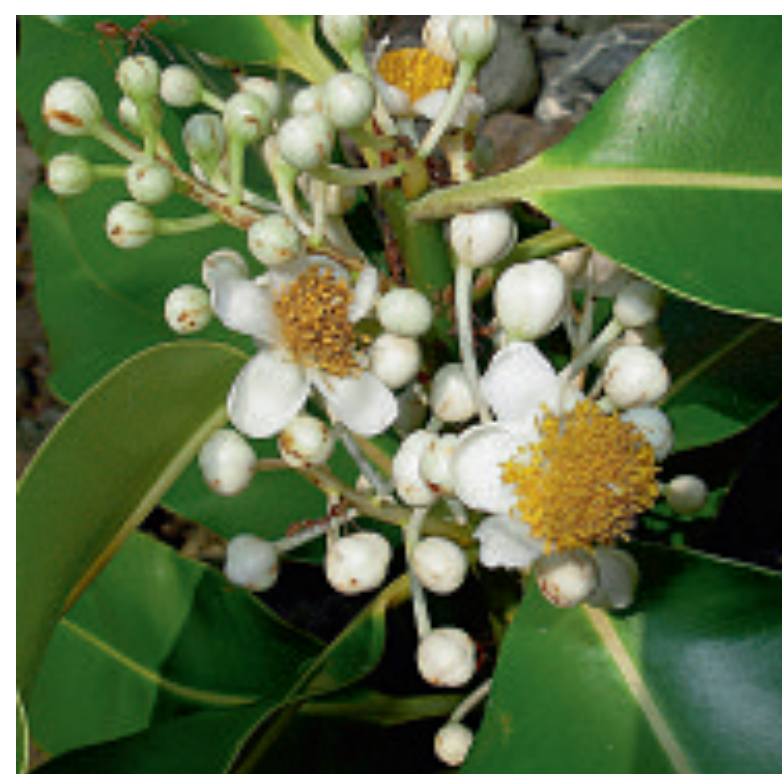




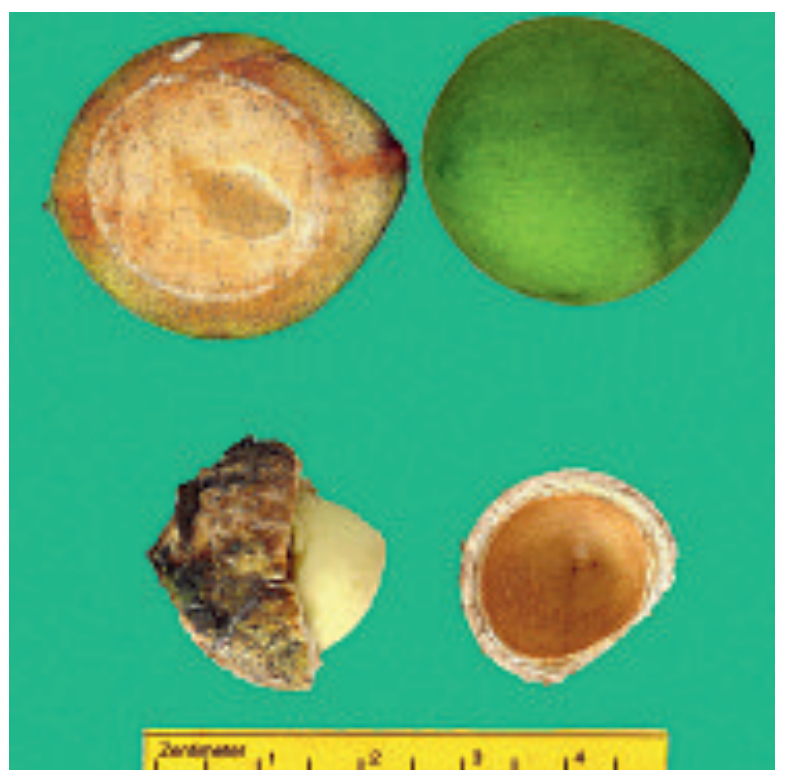

Die Blätter sind meist gegenständig orientiert. Sie sind sattgrün, glänzend und fühlen sich lederartig an. Ihre Form ist elliptisch, länglich oval bis verkehrt eiförmig. Sie sind durchschnittlich zwischen 8 und $20 \mathrm{~cm}$ lang. Ihre Spitze ist rund oder etwas eingebuchtet. Die weißen Blüten erscheinen oft ganzjährig, vermehrt im späten Frühling und späten Herbst. Sie sind in Trauben oder Rispen mit 5-15 Blüten angeordnet und verströmen einen parfümartigen, intensiven Duft, der selbst im getrockneten Zustand anhält. Die Blüten haben einen Durchmesser von $25 \mathrm{~mm}$. Dabei umgeben 4-6 weiße Blütenblätter zahlreiche Staubblätter. Der rosarote Fruchtknoten ist oberständig.

Die Bäume tragen ab einem Alter von ca. 10 Jahren erstmals Früchte. Die grünen Steinfrüchte sind rund oder oval und $25-50 \mathrm{~mm}$ lang. Ihr Kern („Nuss“) ist sehr hartschalig und enthält nur einen Samen. Die äußere Fruchtwand ist in reifem Zustand faltig und gelb bis rotbraun gefärbt. Die weißlichen Samen werden bis 2,5 cm lang (vgl. auch BACKer \& BAKhusen van den Brink 1963, Prosea 1998).

\section{Nutzung}

Das Holz ist sehr dauerhaft und wird schon seit langem genutzt. Es wird im Bootsbau (auch Einbäume) und zur Anfertigung von Möbeln verwendet. Es dient zudem als Parkett, für Türen und Fensterrahmen und als Deckfurnier bei Sperrholz. Sein spezifisches Gewicht wird mit 560 bis $900 \mathrm{~kg} / \mathrm{m}^{3}$ angegeben. Die Samen enthalten 50-74\% Öl, was Calophyllum als Öl liefernde Pflanze interessant macht. Das Öl enthält vor allem die ungesättigten Fettsäuren Ölund Linolsäure, weniger dagegen gesättigte Fettsäuren wie Stearin- und Palmitinsäure. Noch ist Calophyllum als Ölpflanze kaum bekannt und wird in den wenigsten Auflistungen von Ölpflanzen erwähnt.

Das aus den Samen extrahierte Tamanu-Öl kann als Grundlage für Bio-Dieselöl dienen. Ein Liter Tamanu-Öl ergibt einen Liter Bio-Dieselöl. Unbehandelt ist es giftig und nicht für den menschlichen Verzehr geeignet. Ein voll entwickelter Baum kann bei bestem Fruchtansatz bis $100 \mathrm{~kg}$ „Nüsse“ produzieren, aus denen 18,4 kg Tamanu-Öl gewonnen werden kann. Ein Hektar, bestockt mit 400 Bäumen, erbrachte bei einer Vollernte 4680 kg Öl. Das sind im Durchschnitt $11,7 \mathrm{~kg}$ Öl pro Baum. Aus 2,5 kg Samen kann ein Liter Tamanu-Öl gepresst werden. Von 75 getesteten Ölpflanzen erzielte $C$. inophyllum den höchsten Öl-Ertrag pro Baum. Das aus Tamanu-Öl aufgearbeitete Diesel entspricht den deutschen Dieselnormen (DIN V 51606), den Normen der EU (EN 14214) und der USA (ASTM D 6751-02, ASTM PS 121-99).

Chrysophyllum inophyllum ist zudem als Zierbaum sehr beliebt wegen der schönen, glänzenden Blätter und seiner intensiv duftenden Blüten. Der Baum wird in Aufforstungen und besonders zur Erosionsverhütung an sandigen Küsten angepflanzt (Burkhill 1966). Alle Teile des Baumes finden in der Ethnomedizin Verwendung. Kürzlich wurden Inhaltsstoffe entdeckt, die in der Krebs- und HIV-Therapie unterstützend wirken könnten.

\section{Literatur \\ Prosea (Plant Resource of South East Asia) 1998: Lesser known timbers Vol. 5, part 3. - Portland. \\ BACKer, C.A. \& BAKHUIZEN VAN DEN BRINK, R.C. 1963: Flora of Java, Vol.1. - Groningen. \\ Burkhill, L.H. 1966: A dictionary of the economic products of the Malay peninsula Vol. 1. - Kuala Lumpur.}

Abb. 2: Geöffnete Frucht mit Samen. 